\title{
Féeries
}

Études sur le conte merveilleuX, XVII $-\mathrm{XIX}{ }^{\mathrm{e}}$ siècle

$10 \mid 2013$

Conte et croyance

\section{Henri de Montfaucon de Villars, Le Comte de Gabalis, ou Entretiens sur les sciences secrètes}

(avec l'adaptation du Liber de Nymphis de Paracelse par Blaise de Vigenère, 1583), édition présentée et annotée par Didier Kahn, Paris, Honoré Champion, « Sources classiques, n 105 », 2010, 320 p.

Jean-François Perrin

\section{(2) OpenEdition}

\section{Édition électronique}

URL : http://journals.openedition.org/feeries/895

DOI : 10.4000/feeries.895

ISSN : 1957-7753

Éditeur

UGA Éditions/Université Grenoble Alpes

\section{Édition imprimée}

Date de publication : 20 septembre 2013

Pagination : $273-278$

ISBN : 978-2-84310-253-0

ISSN : $1766-2842$

\section{Référence électronique}

Jean-François Perrin, « Henri de Montfaucon de Villars, Le Comte de Gabalis, ou Entretiens sur les sciences secrètes », Féeries [En ligne], 10 | 2013, mis en ligne le 20 mars 2015, consulté le 22 septembre 2020. URL : http://journals.openedition.org/feeries/895; DOI : https://doi.org/10.4000/ feeries.895

\section{(c) Féeries}




\section{COMPTES RENDUS CRITIQUES}

Le Comte de Gabalis, ou Entretiens sur les sciences secrètes. (Avec l'adaptation du Liber de Nymphis de Paracelse par Blaise de Vigenère, I583), Henri de Montfaucon de Villars, édition présentée et annotée par Didier Kahn, Paris, Honoré Champion, «Sources classiques», $\mathrm{n}^{\circ}$ I05, 320 p., 2010.

Venant à la suite de l'excellente édition de Roger Laufer (Nizet, 1963), l'apport de cette édition est considérable. Il est de trois ordres : une connaissance plus précise et mieux contextualisée de la vie et des activités littéraires de l'abbé de Villars; une documentation renouvelée des sources bibliques, savantes ou littéraires du Comte de Gabalis; une approche neuve de son dialogisme d'après certaines œuvres contemporaines, notamment les Provinciales. On aura cependant quelque réserve sur certains aspects de la présentation des enjeux littéraires de cette brillante fiction dialogique.

Après une vaste et riche introduction sur laquelle je vais revenir, le texte est édité dans l'édition originale de I670 (ce qui avait été le choix de R. Laufer), mais en orthographe modernisée, la ponctuation d'origine étant maintenue. Il a été tenu compte de quelques nouveaux éléments touchant l'établissement du texte apparus depuis l'édition Laufer. La riche annotation au fil des pages éclaire considérablement les sources, emprunts, plagiats où Villars a puisé sa matière. On trouve en annexe des documents éclairant certaines allusions du texte : L'Horoscope du Christ par Jérôme Cardan dont une introduction substantielle précise la transmission vers Villars par la lecture probable de Vanini; le récit de la visite du père de Cardan par des êtres surnaturels, un commentaire du démonologue Jean Wier sur la question de la chute des anges, des extraits significatifs des emprunts de Villars à un autre démonologue : Martin Del Rio, et enfin deux textes de Blaise de Vigenère et de Tite-Live sur les origines légendaires de Servius Tullius.

Le premier chapitre : "Comment naissent les légendes» pointe avec une érudition redoutable tout ce qu'on ignore de l'abbé de Villars malgré 
le peu qu'on croyait en savoir, en ajoutant néanmoins au passage quelques éléments nouveaux survenus depuis l'édition de Laufer, par l'entremise surtout de Jean Lesaulnier et Antony McKenna. On apprend donc qu'un libraire-éditeur du XIX ${ }^{\mathrm{e}}$ siècle, René-Louis Doyon, en rééditant le Comte de Gabalis, a doté son auteur de trois prénoms qui restent encore les siens au catalogue de la BNF, alors qu'Henri est le seul attesté. Quant aux démêlés déjà connus de Villars avec la justice toulousaine (il est condamné en I669 avec ses deux frères pour assassinat et incendie du bien d'autrui — la fuite les tire d'affaire), ils en font un aventurier des Lettres comme il y en eut de Gatien de Courtilz à Casanova; Roger Laufer l'avait signalé dans son édition : il s'agissait d'une sombre vendetta familiale remontant à I662. La suite est parisienne : Didier Kahn nous apprend que Villars a fréquenté l'académie de l'abbé d'Aubignac où il pouvait rencontrer des érudits un peu libertins, et qu'il était reçu chez quelques grands noms: Hugues de Lionne, Michel Le Tellier. Il paraît avoir eu des contacts avec Port-Royal par un cousin germain, mais ne tardera guère à s'attirer les foudres d'Arnauld avec la publication du Comte de Gabalis qui lui vaut interdiction de chaire et le discrédite dans l'aristocratie jansénisante. On découvre alors le rôle joué par le De la délicatesse de Villars (I67I) dans la polémique de Bouhours avec les jansénistes sur la question du style, dans ses Entretiens d'Ariste et d'Eugène (I67I). Si le premier l'en remercie publiquement, ses satires lui valent l'ire des seconds, ce qui explique peut-être l'émersion dans l'imprimé, en I671, de l'arrêt toulousain de I669, muni d'un "chapeau» précisant que «l'auteur du Comte de Gabalis, \& de la Delicatesse... a esté condamné avec ses complices à estre rompu tout vif, \& à expirer sur la rouë, \& leurs biens confisquez pour crimes d'assassin, meurtre $\&$ incendie» (document joint en annexe, p. 34-37, suivi d'une généalogie toulousaine de la famille de Montfaucon).

Après quoi, le silence. On savait qu'il était mort assassiné sur la route de Lyon, mais sans pouvoir préciser date, ni circonstances, ni mobile. Une lettre d'une de ses sœurs récemment découverte, ainsi qu'une autre du père François Oudin semblent indiquer qu'il est tombé, fin 1673, des suites de la vendetta familiale qu'on a vue. Il reste sans doute à écrire, sur cet abbé très singulier, une «histoire tragique» façon Rosset.

Pour ce qui touche la documentation des sources du Comte de Gabalis, il faut souligner l'ampleur de l'apport des recherches de Didier Kahn, qui a tiré le meilleur parti de celles de Laufer (rarement pris en défaut ici), même s'il n'avait pas cité in extenso tous les textes-sources, alors que c'est le choix — fort précieux pour le chercheur! — de l'annotation de la présente édition. L'avantage de la méthode de $\mathrm{D}$. Kahn est qu'on prend tout à fait conscience 
du caractère de montage ou de marqueterie littéraire que revêt le texte de Villars : il s'agit d'un dispositif fictionnel de mise en rapport d'énoncés où le partage apparent entre le discours du "fou" (le Comte) et celui de son interlocuteur (le narrateur faux naif et vrai persifleur) en recouvre d'autres dont le jeu exige du lecteur un travail complexe d'interprétation.

L'éditeur est un spécialiste de l'histoire de l'alchimie et de Paracelse (il a publié un Alchimie et paracelsime en France à la fin de la Renaissance, Droz, 2007) et il participe aux travaux du collectif «Chrysopœia» dans le cadre duquel il a par exemple publié les actes du Ir colloque international de la Société d'Étude de l'Histoire de l'Alchimie, Alchimie : art, histoire et mythes (SÉHA-Archè, Paris-Milan, 1995). Sa compétence et ses recherches dans ce domaine nourrissent évidemment son édition. Le chapitre 2 de son introduction fournit donc d'utiles et nécessaires mises au point sur un domaine qu'on connaît rarement de première main, encore moins selon une approche historienne authentique, et à propos duquel mythes et idées fausses vont évidemment leur train ordinaire. Que désigne-t-on au juste par «sciences secrètes» au XVII ${ }^{\mathrm{e}}$ siècle? À quelles disciplines distinctes ce fourre-tout renvoie-t-il ? Quel crédit leur reste-t-il pour des esprits devenus cartésiens, mais aussi pour ceux qu'occupe l'effrayante affaire des poisons? Qui est Paracelse? Quel est encore l'impact de ses idées médico-philosophiques et alchimistes à la fin du XVII ${ }^{\mathrm{e}}$ siècle? Ces questions trouvent ici des réponses tout à fait précises et même fort détaillées parfois. Qui voudrait se faire une idée de l'état scientifique des connaissances concernant la présence de l'alchimie, de la magie, de la géomancie, de l'astrologie, de la cabale, dans l'histoire culturelle de cette époque depuis la Renaissance, trouvera ample matière à satisfaire sa curiosité dans ce chapitre aussi dense que clair. Il en comprendra d'autant mieux le degré de familiarité de Villars et de son époque avec ces disciplines autant qu'avec leurs adversaires. Plus anecdotiquement, il trouvera aussi, au fil, d'utiles mises au point lexicales, comme par exemple, au chapitre 4, sur le nom de Gabalis « compagnon des anges" selon la Basilica Chymica d'Oswald Croll (I609) mais aussi dérivé de gabalus, potence, gibet... gibier de potence (p. 88).

Mais ce n'est pas tout : D. Kahn montre que si Montfaucon de Villars a puisé chez Paracelse l'essentiel de la théorie des esprits élémentaires (ce qu'on savait), ce n'est pas directement dans ses Opera omnia (alors rééditées à Genève et disponibles en France), mais par une source intermédiaire : l'adaptation du Liber de nymphis, sylphis, pigmoeis et salamandris paracelsien par Blaise de Vigenère, accessible dans les notes de sa traduction de la première décade de Tite-Live (1583), rééditée en I6I6-I6I7; Vigenère y réorganise et abrège le traité de Paracelse sans défigurer ses thèses. Au chapitre 4 de son 
introduction, D. Kahn indique dans quel esprit il le fait et démontre que Villars a procédé à de très nombreux collages textuels directement puisés dans cette adaptation, quoique sans la suivre partout, soit qu'il l'adapte lui-même aux besoins de sa propre entreprise, soit qu'il la complète en recourant, cette fois directement dans le Liber de nymphis, à des éléments négligés par Vigenère. Il s'agit, autrement dit, d'une adaptation recréatrice de l'adaptation de Vigenère, laquelle est offerte au lecteur en édition critique aux p. 98-II3. On la retrouve également dans l'annotation du texte.

Outre cette découverte, D. Kahn a systématiquement dépouillé les prélèvements-montages opérés par Villars dans des sources qu'avait en partie repérées Laufer sans les exploiter à fond : la démonologie avec Jean Wier, Jean Bodin, Martin Del Rio; l'humanisme de la Renaissance : Marsile Ficin, Agostino Steuco, L. G. Gyraldi; les sources bibliques de Villars (le discours du comte cabaliste est farci de citations authentiques ou détournées de l'Ancien Testament ou de l'Évangile) qui sont abondamment précisées dans l'annotation du texte, de concert avec ce qui revient à Lulle, Agrippa et quelques autres; enfin, l'averroïsme padouan et le libertinage savant via Cardan, Vanini et Naudé (pour l'Apologie des grands hommes accusez de magie), et Cyrano de Bergerac, qui trouvent une place de choix dans cette édition, non seulement en termes de sources mais aussi parce que D. Kahn interprète la visée de Villars comme "projet libertin» dans son chapitre 2, que ce soit dans le travail littéral avec les textes de Vanini et Naudé, l'effacement du salut par le Christ en faveur de la médiation sylphique qui rend accès à la pureté adamique perdue, ou encore dans la construction même des Entretiens (p. 70-7I). La thèse est bien défendue; je crois tout de même moins à l'existence d'un projet délibéré qu'à l'expression d'un tempérament foncièrement douteur ou sceptique à l'égard du jeu et des règles du jeu dialectique, comme avec Montaigne et, dans une certaine mesure, La Fontaine : cette fiction, qui se donne comme telle ouvertement, refuse de livrer une clef d'interprétation claire et joue sur des ambiguïtés savamment construites puisque, en dépit de la manie interprétative du comte qui finit toujours par tout ramener à sa grille paracelsienne, les deux interlocuteurs partagent en réalité un terrain d'approche rationaliste de la croyance, autant qu'un corpus de référence chrétien. Tout est fait pour inquiéter le lecteur à ce sujet, pour le laisser par lui-même s'en dépêtrer selon son propre fonds de croyances.

Sur le plan des formes littéraires, D. Kahn indique au chapitre 4 de son introduction l'impact possible de Sendivogius, du Vitulus aureus (I667) de Jean-Frédéric Helvétius et des Rares expériences (I668) de Moras de Respour, dans l'idée de départ du Comte de Gabalis, que ce soit pour le personnage du comte ou le genre de l'entretien sur les sciences secrètes. On reste ici 
dans le domaine de la conjecture relativement prudente. Plus persuasive est la suggestion, au chapitre 5, d'une influence du style de Pascal dans les Provinciales sur le dialogisme du Comte de Gabalis — influence déjà signalée par Philippe Sellier dont D. Kahn suit ici la leçon : pour amener le comte aux dernières extrémités de son délire, le narrateur imite en effet la posture de Montalte vis à vis de son jésuite, à la fois attentif, doucement provocateur et aimablement persifleur; en revanche, il y aurait à nuancer beaucoup sur le personnage de Gabalis lui-même, qui est bien plus intéressant, comme caractère, que la cible de Pascal : aussi sage que fou selon les matières dont il parle, il dispose d'une solide culture chrétienne, sait l'employer à propos dans le débat, et partage à peu près les mêmes idées que son interlocuteur sur la superstition. Il est à certains égards comparable à Don Quichotte : il n'est fou que dans sa manie de tout réinterpréter in fine en faveur de son système.

Il est bien regrettable à cet égard que D. Kahn n'ait pas lu les pages consacrées par Jean-Paul Sermain au Comte de Gabalis dans son Métafictions (Champion, 2002), ouvrage entièrement consacré aux mutations du roman et de la fiction dans les années 1670-1730. Le dispositif inventé par Villars : mise en scène dialoguée/dramatisée de la croyance traitée selon le point de vue de l'illusionné dans une fiction qui s'avoue telle et laisse au lecteur le soin de se faire sa propre idée des enjeux en discussion, est la clef aussi bien de la poétique du merveilleux dans le conte de fées, comme le savent les lecteurs de Féeries, que de l'esthétique du roman moderne tel qu'il va se développer dans les années 30 du XVIII ${ }^{\mathrm{e}}$ siècle français, en particulier dans le choix de faire fiction des matières de réflexion. Le succès du Comte de Gabalis s'inscrit précisément à cet endroit de l'histoire des formes et des mentalités, comme cristallisation géniale d'une mutation en cours... tout près des Amours de Psyché de La Fontaine qui, lui aussi, mais en pleine conscience d'artiste si l'on en juge par sa préface, voit venir l'esprit moderne et cherche une forme apte à lui plaire sans renoncer au grand art classique. Il y a un bouleversement des poétiques et des genres à travers l'épreuve de la Querelle des Anciens et des Modernes, qui transforme l'univers des fictions en un véritable chantier d'invention de formes neuves : des Entretiens sur les sciences secrètes au Neveu de Rameau, il existe bel et bien une parenté profonde d'esprit et de technique, comme le signalait d'ailleurs déjà l'introduction littéraire de l'édition Laufer qui, sur bien des points, mérite encore amplement d'être relue.

Ces réserves faites, il faut confirmer la richesse de l'édition de D. Kahn. Son annotation et toute la documentation savamment présentée dans son introduction accentuent et raffinent encore le plaisir qu'on prend à se 
plonger dans ces débats d'outre-temps traités dans une langue vraiment extraordinaire d'à-propos et de finesse désinvolte.

Jean-François Perrin

Le Problème de l'incroyance au XVIII siècle, Paul Pelckmans, Les Presses de l'université Laval, "Cahiers du CIERL», I6o p., 20 Io.

Ce petit livre est un grand livre. Il s'inscrit dans l'œuvre considérable de Paul Pelckmans et vient compléter une série d'enquêtes qui abordent l'histoire des mentalités et reconstituent comme une anthropologie des consciences en prenant appui sur les textes littéraires, depuis sa thèse sur Le Sacre $d u$ père, Fictions des Lumières et historicité d'Edipe I699-I775 (Rodopi, I983) jusqu'à Écrire l'insignifiant (2000) et Concurrence au monde, Proposition pour une poétique du collectionneur moderne (2004) (à côté d'ouvrages consacrés à Cleveland, à Werther, à Isabelle de Charrière). Petit ce livre l'est par son format, c'est nous dit l'auteur «un essai» (de I59 pages + XXII) : il se refuse à effectuer des enquêtes exhaustives qui iraient se perdre dans les plus obscurs recoins avec l'espoir d'y trouver des perles ensevelies dans la poussière et inconsciemment oubliées. Il se contente d'une série d'exemples qu'il observe avec humilité : il s'agit pour lui de relever les traces d'une croyance religieuse (d'inspiration catholique). Pour cela il envisage la foi sous plusieurs aspects et dans des textes de genres variés et de nombreux auteurs. Ainsi voit-il tout d'abord sous le titre de "providences romanesques» comment Mouhy et Prévost laissent leurs personnages afficher une soumission à l'ordre divin. Puis il poursuit la même enquête chez les philosophes. Un chapitre est consacré à Zaïre de Voltaire et à l'élévation sur la Sainte Croix. Un autre intéresse Diderot dans les Salons et la manière dont il traite les toiles de sujet religieux (avec des regards sur La Religieuse). P. Pelckmans examine enfin comment les personnages pratiquent les sacrements : baptême, confessions, liturgies de la mort. Dans chaque cas, il prend en compte le point de vue du personnage, le contexte du passage pour remonter au point de vue de l'auteur : il cherche à distinguer ce qui relève d'habitus d'une société substantiellement définie par son adhésion religieuse, de formules ou en quelque sorte d'obligations culturelles; il tente d'évaluer les gestes religieux en confrontant leur caractérisation, leur rôle dans l'œuvre littéraire avec leur signification au sein de la tradition catholique (pour l'essentiel). Si la démarche est ainsi pédestre, le regard de P. Pelckmans est ample (passant d'un domaine à l'autre, d'un

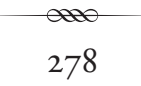

